\title{
Optimal time to surgery following neoadjuvant chemotherapy and its effect on the survival of patients with breast cancer
}

\author{
B. SRINIVAS PEDAMALLU ${ }^{1}$, DELILAH HASSANALLY ${ }^{2}$, ABDUL KASEM ${ }^{1}$, \\ ${\text { CHARLOTTE } \mathrm{ABSON}^{3} \text { and IBRAHIM AHMED }}^{2}$ \\ ${ }^{1}$ Department of Oncoplastic Breast Surgery, Kings College Hospitals NHS Trust, Princess Royal University Hospital, \\ Orpington BR6 8ND; Departments of ${ }^{2}$ Oncoplastic Breast Surgery, and ${ }^{3}$ Medical Oncology, \\ Medway Hospitals NHS Trust, Medway Hospital, Gillingham, Kent ME7 5NY, UK
}

Received September 27, 2021; Accepted December 9, 2021

DOI: $10.3892 /$ wasj.2021.140

\begin{abstract}
There is currently an ongoing debate regarding the time to surgery (TS) following neoadjuvant chemotherapy in patients with breast cancer and its effect on survival outcomes. There are three retrospective studies suggesting that the ideal interval is as low as 21 days, 40 days and up to 8 weeks. To date, to the best of our knowledge, there is no consensus available on the ideal time interval to surgery following neoadjuvant chemotherapy in breast cancer patients in the published literature. The present study aimed to evaluate the influence of TS of $\geq 28$ days and its effect on survival outcomes. For this purpose, patients with breast cancer $(n=61)$, during the time period between January, 2012 and December, 2016, were categorised into two cohorts based on the TS following the completion of neoadjuvant chemotherapy. Patients in group $1(n=8)$ had a TS of $\leq 28$ days, and those in group 2 had a TS of $\geq 29$ days $(n=53)$. Overall survival and locoregional recurrence-free survival were compared between both groups. A total of 61 patients with breast cancer who had received neoadjuvant chemotherapy followed by surgery and fulfilled the inclusion criteria were included in the study. The median follow-up time was 29 months. There was no observed association between age, tumour biology, the chemotherapy regimen, type of surgery, pathological response and the TS. The mortality rates were zero in group 1 and $18.9 \%$ in group 2. Locoregional recurrence rates were zero in group 1 and $9.4 \%$ in group 2. On the whole, the findings of the present study support a shorter duration ( $\leq 28$ days) of TS following neoadjuvant chemotherapy for optimal survival outcomes.
\end{abstract}

Correspondence to: Dr B. Srinivas Pedamallu, Department of Oncoplastic Breast Surgery, Kings College Hospitals NHS Trust, Princess Royal University Hospital, Farnborough Common, Orpington BR6 8ND, UK

E-mail: pbs6vas@yahoo.com

Key words: neoadjuvant chemotherapy, time to surgery, overall survival, locoregional recurrence-free survival, breast cancer, survival outcomes

\section{Introduction}

Neoadjuvant chemotherapy for the treatment of breast cancer is well-established as a therapeutic modality for locally advanced disease, inflammatory cancers, large tumours, for selected high-risk cases and for patients who may otherwise require a mastectomy due to the small size of the breast or the position of the tumour within it. The use of neoadjuvant chemotherapy provides patients with large tumours, the possibility of surgical resection or breast-conserving surgery rather than mastectomy due to the shrinkage of the tumour. Neoadjuvant chemotherapy allows for the active monitoring of the response and the discontinuation of inactive therapy in the event of disease progression.

The optimal timing for neoadjuvant chemotherapy in breast cancer has long been debated and studied. The optimal time interval between the end of neoadjuvant chemotherapy and definitive surgery remains unclear. However, some large randomized clinical trials have demonstrated no significant differences in disease-free and overall survival between patients receiving chemotherapy in the adjuvant and the neoadjuvant settings (1-4).

Some published studies have investigated the proper timing for the initiation of adjuvant chemotherapy following surgery in patients with breast cancer. Available data from these trials revealed that there is a decrease in the efficacy of adjuvant systemic therapy when the delay was $>12$ weeks post-surgery $(3,4)$. The Current European Society of Medical Oncology (ESMO) guidelines suggest that systemic adjuvant treatment should preferably commence within 2-6 weeks following surgery (5). However, no recommendation regarding the most effective time interval between neoadjuvant chemotherapy and surgery has yet been made.

To date, at least to the best of our knowledge, no large randomized control trials on neoadjuvant systemic therapy have addressed the issue associated with the delays in time to surgery (TS) following the completion of neoadjuvant chemotherapy and its effect on survival outcomes.

The increasing need for multidisciplinary input, genetic testing and counselling for reconstructive procedures, including contralateral prophylactic surgery is known to contribute to delays in these high-risk patients. The general 
consensus is to perform surgery as soon as possible, when the neutropenic window is overcome and following the resolution of any short-term chemotherapy toxicities. Surgical intervention is typically performed within 4-6 weeks following the completion of neoadjuvant chemotherapy. This allows patients to recover sufficiently from the side-effects of neoadjuvant chemotherapy, and theoretically prevents potential tumour regrowth. However, the acceptable maximum time interval is yet unknown.

Prolonged intervals without exposure to chemotherapy and without surgical intervention may allow tumour neo-angiogenesis, tumour growth and a possible theoretical increased risk of recurrence. A more effective response to neoadjuvant chemotherapy predicts decreased rates of recurrence and an improved overall survival rate. In addition, it was described in the literature that significant reductions in $\mathrm{Ki}-67$ expression levels following neoadjuvant chemotherapy may be associated with decreased recurrence rates.

The timing of surgery following neoadjuvant chemotherapy is crucial and three previous studies have investigated its effect on the survival outcomes of these high-risk patients. Sanford et al (6) demonstrated that patients with neoadjuvant chemotherapy to surgery intervals of up to 8 weeks had equivalent overall, recurrence-free and locoregional recurrence-free survival rates. Another published study suggested that significantly worse overall and recurrence-free survival rates were associated with a TS >21 days (7). In addition, it has been reported that patients who had undergone surgery $>40$ days following neoadjuvant chemotherapy had lesser reductions in Ki-67 levels, potentially indicating tumour regrowth and predicting a worse oncological outcome (8).

As per the authors' observation, the delays were mainly due to chemotherapy side-effects, post-chemotherapy scans, delayed multi-disciplinary team (MDT) discussion, clinical appointments, long waiting lists and patient's decision to postpone surgery. Unnecessary delays can affect the survival outcomes of these high-risk patients. The Breast Unit Multidisciplinary team at Medway Hospital is in agreement that an ideal interval from neoadjuvant chemotherapy to surgery of $\leq 28$ days or less would allow a reasonable time to recover from side-effects and would also allow adequate time for surgical planning, yet not compromising the survival outcomes. Thus, the present study aimed to evaluate the efficacy of TS of $\geq 28$ days in post-neoadjuvant chemotherapy breast cancer patients and to determine its effect on survival outcomes.

\section{Patients and methods}

The present study was retrospective study on female patients who had received neoadjuvant chemotherapy followed by breast-conserving surgery or mastectomy for breast cancer (inclusion criteria) between January 1, 2012 and December 31, 2016 at Medway Hospital, Gillingham, Kent, UK. Patients with recurrent breast cancer, those with distant metastases and male patients were excluded from the study. UK Research Ethics Committees (RECs) and HRA approval for the present study was obtained (REC reference: 17/NS/0007 and IRAS project ID: 222885).

A total of 61 breast cancer patients who had received neoadjuvant chemotherapy fulfilled the inclusion and exclusion criteria. The data elements extracted were patient age, tumour type, grade, hormone receptor status, HER2 status, lymphovascular invasion and evidence of lymph node involvement, the time interval between the completion of the last cycle of neoadjuvant chemotherapy to surgery, any evidence of local or regional recurrence in the post-operative follow-up period, evidence of distant metastasis in the post-operative follow-up period and mortality in the post-operative follow-up period.

These patients were categorised into two cohorts based on the TS following the completion of neoadjuvant chemotherapy based on the multidisciplinary team agreement of the Breast Unit of Medway Hospital on the ideal TS. The patients in group $1(n=8)$ had a TS of $\leq 28$ days, and those in group 2 had a TS of $\geq 29$ days $(n=53)$. Overall and locoregional recurrence-free survival were compared between both groups. Locoregional recurrence-free and overall survival curves were constructed using the Kaplan-Meier method for both cohorts and P-values calculated.

Statistical analysis. The overall survival and locoregional recurrence-free survival of the patient cohorts was analysed using Kaplan-Meier analysis with the log-rank test for statistical significance. A P-value $<0.05$ was considered to indicate a statistically significant difference.

\section{Results}

Details of age distribution, the type of breast cancer, receptor positivity and type of surgery performed in the study groups are presented in Table I. A total of 61 breast cancer patients who had received neoadjuvant chemotherapy and satisfied the inclusion criteria were included in the study. In total, $13.1 \%$ of the patients in the study group $(n=8)$ had a TS of $\leq 28$ days and comprised group 1 and $86.9 \%$ of the patients $(n=53)$ with a TS of $\geq 29$ days comprised group 2 . The rate of breast-conserving surgery in the study group was $62 \%$, with $100 \%$ of the patients in group 1 and $56 \%$ in group 2, respectively. The follow-up period was 13-73 months. The median follow-up time was 29 months. The TS and patient characteristics including age, nuclear grade, histology, lymphovascular invasion, tumour biology (HER2- positive, triple-negative, or hormone-receptor status), the presence of pathological complete response, and surgery type (breast-conserving surgery or mastectomy) were not compared between the two groups, as there were no observed differences.

The Kaplan-Meier estimates of survival in both cohorts were calculated for overall survival and locoregional recurrence-free survival. A P-value of 0.17 was estimated for overall survival with no statistically significant difference (Fig. 1). The estimated P-value for locoregional recurrence-free survival was 0.36 with no statistically significant difference (Fig. 2). The mortality rates were zero in group 1 and $18.9 \%$ in group 2 . Locoregional recurrence rates were zero in group 1 and $9.4 \%$ in group 2 (Fig. 3).

\section{Discussion}

The optimal time interval between the completion of neoadjuvant chemotherapy and definitive surgery in breast cancer 
Table I. Details of patient age distribution, type of breast cancer, receptor positivity and type of surgery performed.

\begin{tabular}{|c|c|}
\hline Parameter & No. of patients \\
\hline \multicolumn{2}{|l|}{ Age groups } \\
\hline $31-50$ years & 33 \\
\hline $51-70$ years & 24 \\
\hline $71-80$ years & 4 \\
\hline \multicolumn{2}{|l|}{ Tumour type } \\
\hline Invasive ductal carcinoma & 55 \\
\hline Invasive lobular carcinoma & 5 \\
\hline Basal type & 1 \\
\hline \multicolumn{2}{|l|}{ Tumour grade } \\
\hline Grade 1 & 0 \\
\hline Grade 2 & 19 \\
\hline Grade 3 & 42 \\
\hline \multicolumn{2}{|l|}{ Receptor status (ER, PR, HER2) } \\
\hline Triple-negative (ER'- $\left.\mathrm{PR}^{-}, \mathrm{HER} 2^{-}\right)$ & 22 \\
\hline $\mathrm{ER}^{+}, \mathrm{PR}^{+}, \mathrm{HER} 2^{-}$ & 11 \\
\hline HER2 only positive $\left(\mathrm{ER}^{-}, \mathrm{PR}^{-}, \mathrm{HER} 2^{+}\right)$ & 12 \\
\hline $\mathrm{ER}^{+}, \mathrm{PR}^{-}, \mathrm{HER}^{+}$ & 3 \\
\hline ER only positive $\left(\mathrm{ER}^{+}, \mathrm{PR}^{-}, \mathrm{HER} 2^{-}\right)$ & 9 \\
\hline Triple-positive $\left(\mathrm{ER}^{+}, \mathrm{PR}^{+}, \mathrm{HER} 2^{+}\right)$ & 4 \\
\hline \multicolumn{2}{|l|}{ Type of surgery performed } \\
\hline Mastectomy & 23 \\
\hline Wide local excision with no wire localisation & 14 \\
\hline Wide local excision with wire localisation & 24 \\
\hline
\end{tabular}

ER, oestrogen receptor; PR, progesterone receptor; HER2, human epidermal growth factor receptor 2 .

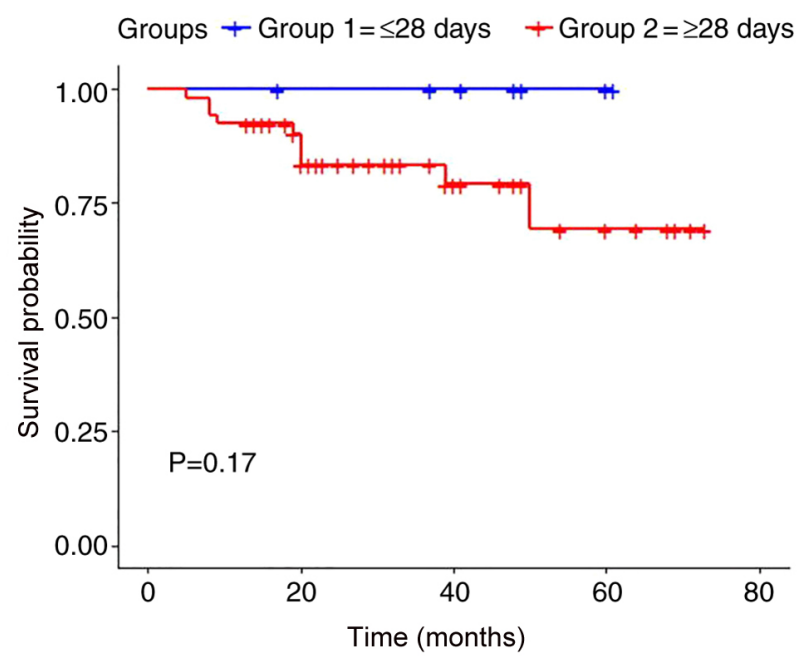

Figure 1. Kaplan-Meier estimates illustrating overall survival curves.

patients remains unclear. To date, no large randomized clinical trials on neoadjuvant systemic therapy have addressed the TS delays and the effect on survival outcomes in patients receiving neoadjuvant chemotherapy. The general consensus is to perform

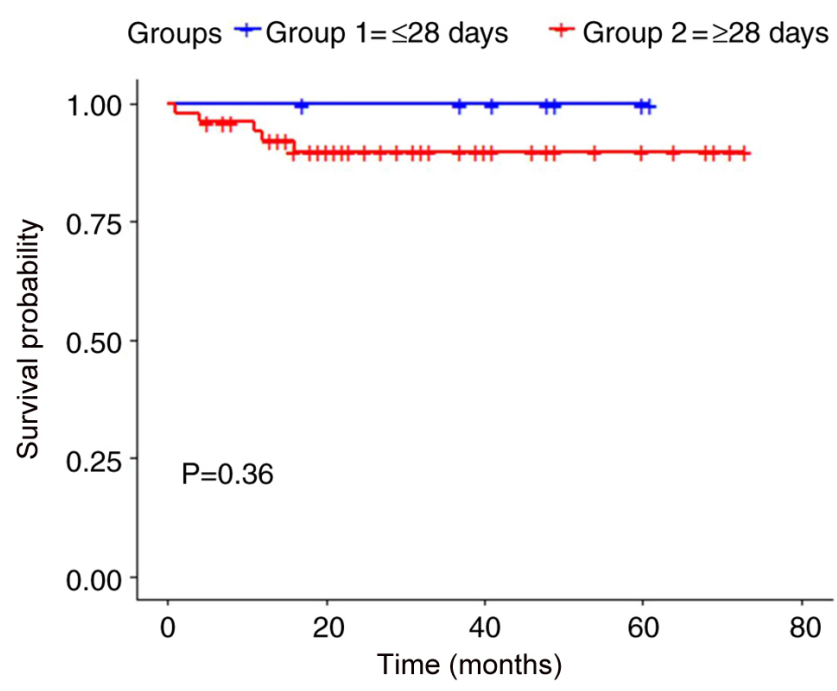

Figure 2. Kaplan-Meier estimates illustrating locoregional recurrence-free survival curves.

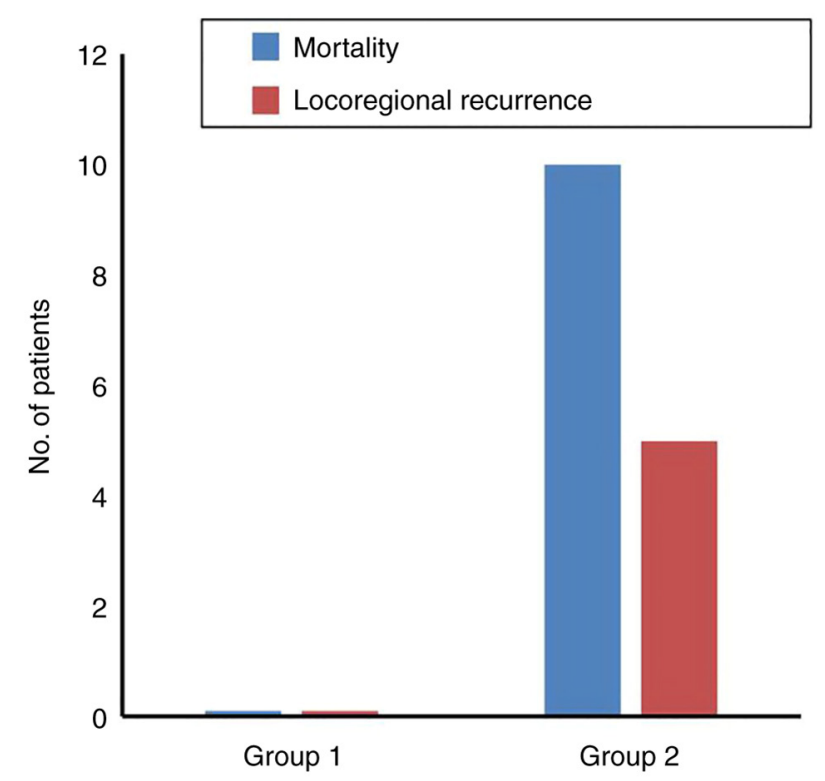

Figure 3. Mortality and recurrence rates of the patients in the two groups. Group $1(n=8)$, patients with a time to surgery of $\leq 28$ days; group $2(n=53)$, patients with a time to surgery of $\geq 29$ days.

surgery following the resolution of chemotherapy-induced neutropenia and short-term chemotherapy-related toxicities.

Omarini et al (7) evaluated the association between TS following neoadjuvant chemotherapy and survival outcomes. They concluded that the patients with breast cancer who underwent surgery within 21 days experienced maximal benefit from the previous treatment and this advantage was consistent and maintained over time. Sanford et al (6) investigated the association between the time interval from neoadjuvant chemotherapy to surgery and survival outcomes at the MD Anderson Centre in the time period between years 1995-2007. They suggested that those patients with neoadjuvant chemotherapy to surgery intervals of up to 8 weeks had equivalent overall, recurrence-free and locoregional recurrence-free survival rates. 
Prolonged TS intervals may theoretically increase the risk of recurrence by allowing tumour neo-angiogenesis and tumour growth. Gabordi et al (8) evaluated the association between TS from the completion of neoadjuvant chemotherapy and the reduction in $\mathrm{Ki}-67$ levels. They reported that patients who underwent surgery within 40 days from the completion of neoadjuvant chemotherapy experienced a greater reduction in Ki-67 levels. Patients undergoing surgical intervention $>40$ days following neoadjuvant chemotherapy had lesser reductions in Ki-67, potentially indicating tumour regrowth and predicting a worse oncologic outcome.

The breast multidisciplinary team at Medway Hospital is in agreement that an ideal TS interval of $\leq 28$ days, allows for a reasonable time to recover from side-effects and allows adequate surgical planning. It is considered $\leq 21$ days is too short to be practically achievable and does not allow sufficient time to recover from the side-effects of chemotherapy.

In the present study, $86.8 \%$ of the patients (group 2) underwent surgery at $\geq 29$ days following the completion of neoadjuvant chemotherapy. In total, $13.1 \%$ of the patients (group 1) had surgery at $\leq 28$ days following the completion of neoadjuvant chemotherapy. Kaplan-Meier estimates of overall survival (P-value of 0.17) and locoregional recurrence-free survival (P-value of 0.36 ) revealed no statistically significant differences. However, a trend towards superior survival outcomes with a TS interval of $\leq 28$ days was observed.

It should be noted however, that the present study has certain limitations due to the small samples size and its retrospective nature.

In conclusion, the analysis of the data in the present study suggests a trend towards favourable survival outcomes following neoadjuvant chemotherapy if the TS is $\leq 28$ days. A target interval of 28 days is achievable and also favours adequate recovery from the side-effects of chemotherapy. The findings of the present study support a shorter TS following neoadjuvant chemotherapy for an optimal survival outcome in these high-risk patients.

\section{Acknowledgements}

The authors would like to extend their special gratitude to Dr Maher Hadaki, Consultant medical oncologist, Medway Hospital for providing access to the trust oncology database and for his valuable assistance during the data collection phase.

\section{Funding}

No funding was received.

\section{Availability of data and materials}

The availability of datasets used and/or analysed during the current study are available only from the corresponding author on reasonable request subjected to prior approval by Medway hospital ethics and clinical governance committee.

\section{Authors' contributions}

BSP was involved in the conception and design of the study, as well as in data collection and data analysis, and in the writing, revising and reviewing of the manuscript. $\mathrm{DH}, \mathrm{AK}, \mathrm{CA}$ and IA were involved in the conception and design of the study, and in the revising and reviewing of the manuscript. BSP and IA confirm the authenticity of all the raw data. All authors have read and approved the final manuscript.

\section{Ethics approval and consent to participate}

NHS Research Ethics Committees (RECs) and HRA approval for the present study was obtained (REC reference: 17/NS/0007 and IRAS project ID: 222885).

\section{Patient consent for publication}

Not applicable.

\section{Competing interests}

The authors declare that they have no competing interests.

\section{References}

1. Deo SV, Bhutani M, Shukla NK, Raina V, Rath GK and Purkayasth J: Randomized trial comparing neo-adjuvant versus adjuvant chemotherapy in operable locally advanced breast cancer (T4b N0-2 M0). J Surg Oncol 84: 192-197, 2003.

2. Mauri D, Pavlidis N and Ioannidis JP: Neoadjuvant versus adjuvant systemic treatment in breast cancer: A meta-analysis. J Natl Cancer Inst 97: 188-194, 2005.

3. Kupstas AR, Hoskin TL, Day CN, Habermann EB and Boughey JC: Effect of surgery type on time to adjuvant chemotherapy and impact of delay on breast cancer survival: A national cancer database analysis. Ann Surg Oncol 26: 3240-3249, 2019.

4. Cai L, Tong Y, Zhu X, Shen K, Zhu J and Chen X: Prolonged time to adjuvant chemotherapy initiation was associated with worse disease outcome in triple negative breast cancer patients. Sci Rep 10: 7029, 2020.

5. European Society for Medical Oncology (ESMO): ESMO Clinical Practice Guidelines: Breast cancer. ESMO, Lugano, 2020. https://www.esmo.org/guidelines/breast-cancer.

6. Sanford RA, Lei X, Barcenas CH, Mittendorf EA, Caudle AS Valero V, Tripathy D, Giordano SH and Chavez-MacGregor M: Impact of time from completion of neoadjuvant chemotherapy to surgery on survival outcomes in breast cancer patients. Ann Surg Oncol 23: 1515-1521, 2016.

7. Omarini C, Guaitoli G, Noventa S, Andreotti A, Gambini A, Palma E, Papi S, Tazzioli G, Balduzzi S, Dominici M, et al: Impact of time to surgery after neoadjuvant chemotherapy in operable breast cancer patients. Eur J Surg Oncol 43: 613-618, 2017.

8. Gabordi RC, Huth J, Rivers A, Hendrix AA, Wooldridge R, Leitch, et al: Optimal time interval to surgery after neoadjuvant chemotherapy in breast cancer. Annual Meeting, The American Society of Breast Surgeons, April 30-May 4, 2014, Las Vegas, NV.

This work is licensed under a Creative Commons Attribution-NonCommercial-NoDerivatives 4.0 International (CC BY-NC-ND 4.0) License. 